\title{
Current and future perspectives on allogeneic transplantation using ex vivo expansion or manipulation of umbilical cord blood cells
}

\author{
Ko K. Maung ${ }^{1}$ (1) $\cdot$ Mitchell E. Horwitz ${ }^{2}$ \\ Received: 3 March 2019 / Revised: 14 May 2019 / Accepted: 15 May 2019 / Published online: 23 May 2019 \\ (c) Japanese Society of Hematology 2019
}

\begin{abstract}
In patients with hematologic malignancies, the outcome of umbilical cord blood transplantation has improved and is now comparable to that of matched unrelated donor transplantation. However, the limitation of using umbilical cord blood has been a delay in both hematopoietic and immunologic recovery. Strategies have been proposed to overcome these limitations. One strategy involves ex vivo expansion of the umbilical cord blood unit prior to transplantation. A second strategy involves exposure of the umbilical cord blood graft to compounds aimed at improving homing and engraftment following transplantation. Many of these strategies are now being tested in late phase multi-center clinical trials. If proven cost effective and efficacious, they may alter the landscape of donor options for allogeneic stem cell transplantation.
\end{abstract}

Keywords Umbilical cord blood · Expansion · Manipulation · Stem cell transplantation · Hematopoietic recovery · Hematopoietic progenitor cell

\section{Introduction}

Umbilical cord blood (UCB) is a highly effective alternative to stem cells collected from healthy adult donors for allogeneic hematopoietic stem cell transplantation. Significant qualitative and quantitative differences exist between the UCB graft and the adult donor graft. Most importantly, the CD34+ hematopoietic stem and progenitor cell doses are significantly lower in the UCB graft. The lower cell dose has the most significant impact on adult recipients, resulting in an increased incidence of graft failure and delayed hematopoietic recovery. While there remains room for improvement, the advent of dual umbilical cord blood

Ko K. Maung

KoKo.Maung@duke.edu

Mitchell E. Horwitz

Mitchell.Horwitz@duke.edu

1 Hematology and Medical Oncology Fellow, Duke University School of Medicine, 2400 Pratt St. DUMC 3961, Durham, NC 27710, USA

2 Adult Blood and Marrow Transplant Program, Duke University School of Medicine, 2400 Pratt St. DUMC 3961, Durham, NC 27710, USA transplantation has reduced the risk of excessive graft failure. Despite the remaining limitations, the outcome of UCB transplantation rivals that of mobilized peripheral blood or bone marrow transplantation from unrelated adult donors [1, 2]. Resolution of the remaining limitations could increase the desirability of the UCB transplantation for adult recipients, perhaps even escalating it to a preferred graft source in patients without a fully matched family member.

Addressing the problem of suboptimal stem cell dose within the UCB graft through ex vivo expansion has met with both practical and technical challenges. Before the advent of dual UCB, cells for ex vivo culture initiation were derived from a small aliquot of the transplanted single UCB graft. With the cell dose already marginal for most adult recipients, this strategy was only appropriate for the limited number of adult recipients with an available UCB unit that exceeded the minimum cell dose requirement. Furthermore, there was no definitive method for assessing the impact of the manipulated cell fraction, particularly the ex vivo expanded hematopoietic stem cell. These hurdles compromised early attempts at ex vivo expansion [3].

New techniques to exploit the proliferative and homing capacity of hematopoietic stem cells in an ex vivo culture system have emerged in recent years. Many of these 
technologies are now being tested in early and late phase clinical trials. If proven safe, effective and practical, these strategies may indeed change the landscape of allogeneic stem cell transplantation.

\section{Hematopoietic stem/progenitor cell manipulation}

Until the advent of dual umbilical cord blood transplantation, the biggest impediment to development of novel hematopoietic stem cell expansion technologies was the ability to safely test and assess the approach in the clinical setting. The emergence of dual UCB transplantation has changed this, paving the way for innovative clinical trials of novel methods of cord blood graft engineering (Table 1) $[4,5]$. As long as the patient receives a single UCB unit of adequate size, a second experimentally manipulated graft can be ethically co-infused and assessed for efficacy. Multiple strategies of ex vivo UCB manipulation have emerged. If lineage committed hematopoietic progenitor cells are targeted for expansion, the period of bone marrow aplasia that complicates intensive chemotherapy can be reduced or eliminated. However, a second, unmanipulated UCB graft containing long-term repopulating hematopoietic stem cells will always need to be co-infused to provide durable hematopoiesis. If long-term repopulating cells are targeted for expansion, the manipulated graft can be expected to provide both short-term and long-term hematopoiesis, thereby eliminating the need for transplantation of a second unmanipulated unit. Another strategy aims not to expand hematopoietic stem/progenitor cells, but to provide them with a homing and engraftment advantage following transplantation. This advantage could both improve kinetics of engraftment as well as reduce the relatively high risk of graft failure that continues to plague the field of UCB transplantation.

\section{Notch-mediated expansion}

Since the early 1990s, the Notch gene family has been implicated in modulation of early hematopoietic stem cell fate [6]. In vitro studies suggested that activation of Notch signaling promotes retention of the most primitive hematopoietic stem cell phenotype during culture. This led investigators at the Fred Hutchinson Cancer Research Center in Seattle Washington to develop a serum-free ex vivo HSC culture system consisting of immobilized Delta1 Notch ligand along with early acting HSC cytokines [stem cell factor (SCF), thrombopoietin (TPO), Flt3 ligand, Il-3 and IL-6] [7]. Of note, cultures were initiated with $\mathrm{CD} 34+$ cells isolated from the UCB unit. The final expanded product contained no T cells.

In the context of a phase I trial using a myeloablative dual umbilical cord blood transplant configuration, patients received one unmanipulated UCB unit and a second unit that was expanded for approximately 16 days in the presence of Notch ligand and cytokines. CD34+ hematopoietic stem-progenitor cells expanded a mean 164-fold during the culture period. In a report describing the outcome of the first 10 patients treated on the trial, patients received an expanded cord blood graft containing an average of $6 \times 10^{6}$ CD34+ cells $/ \mathrm{kg}$ [8]. This compared to $0.24 \times 10^{6}$ from the unmanipulated cord blood graft. The large number of hematopoietic progenitor cells contained within the expanded cord blood graft resulted in a median time to neutrophil engraftment (ANC $>500$ ) of 16 days (range 7-34 days). This compared to 26 days for an historical control population that received standard myeloablative dual cord blood transplantation. Peripheral blood chimerism analysis confirmed that the expanded cord blood unit was responsible for early

Table 1 Strategies for ex vivo manipulation of umbilical cord blood stem cells

\begin{tabular}{lllll}
\hline Compound & Modality & Phase I/II completed (\#pts) & Lead institution/corporate partner & $\begin{array}{c}\text { Active multi- } \\
\text { center phase } \\
\text { II/III }\end{array}$ \\
\hline Notch ligand [8] & Ex vivo expansion & Yes (15) & FHCRC/- & Completed \\
MSC co-culture [24] & Ex vivo expansion & Yes (31) & MDACC/mesoblast & Completed \\
NiCord ${ }^{\circledR}[18,19]$ & Ex vivo expansion & Yes \#1 (11), \#2 (36) & Duke/Gamida cell & Gamida cell/Teva \\
Copper chelation (StemEx) [16] & Ex vivo expansion & Yes (101) & UMinn/Novartis & Completed \\
StemRegenin 1 [26] & Ex vivo expansion & Yes (17) & Maisonneuve-Rosemont Hospital & Completed \\
UM171 [28, 29] & Ex vivo expansion & Yes (25) & DFCI/fate therapeutics & MDACC \\
PGE-2 [31] & HSC modulation & Yes (12) & MDACC/mesoblast & Completed \\
Fucosylation [39, 42] & HSC modulation & Yes (22) & & Ongoing \\
MSC expansion +fucosylation & Ex vivo expan- & Yes (25) & & \\
& sion + HSC modu- & & & \\
\hline
\end{tabular}

FHCRC Fred Hutchinson Cancer Research Center, MDACC MD Anderson Cancer Center, DFCI Dana Farber Cancer Institute 
neutrophil engraftment. However, by 3 months following transplantation, hematopoiesis was derived by the unmanipulated cord blood unit in all but one of the evaluable subjects. While it is possible that transient hematopoiesis from the expanded UCB graft is a consequence of loss of long-term repopulated cells during ex vivo culture, it is more likely that the $\mathrm{T}$ cell replete unmanipulated unit mounted a cellular immune response leading to rejection of the expanded graft $[9,10]$.

Because of the logistical complexity associated with production of a patient-specific expanded cord blood graft, Delaney and colleagues are currently assessing the safety and efficacy of an "off-the-shelf" graft. With this strategy, cord blood transplant recipients receive an unmanipulated single or double UCB transplantation as per standard of care. In addition, the patient receives a fully HLA mismatched third UCB graft that was previously expanded and cryopreserved [11]. The intent is for this mismatched third graft to provide early neutrophil recovery after which it is rejected by the incoming unmanipulated, T-cell replete UCB graft. In a single-center phase II trial at Fred Hutchinson Cancer Center (Clinicaltrials.gov NCT01175785), 15 patients with hematologic malignancies were enrolled from 2010 to 2012 to receive the ex vivo expanded cord blood-derived hematopoietic stem cells using Notch ligand (product was called NLA101). The outcomes were compared to a concurrent control cohort of 50 patients receiving a standard of care cord blood transplant. The median CD34+ and total nucleated cell doses in the study group were 5.3 (range $3.1-11.6) \times 10^{6}$ cells $/ \mathrm{kg}$ and 5.8 (range $\left.2.2-10.9\right) \times 10^{7}$ cells/ $\mathrm{kg}$, respectively. Median neutrophil recovery was 19 (range 9-31) days in study group, compared with 25 (range 14-45) days in the control. Median platelet recovery was 35 (range 21-86) days as compared with 48 (24-158) days in the control group. No patients in study group had grade III-IV acute GvHD compared with $29 \%$ in the control group. At 5 years, 27 of the NLA101 recipients experienced chronic GVHD vs. $38 \%$ of the control group. No patients in the study group experienced transplant-related mortality when compared with $16 \%$ of the control group. Disease-free survival and overall survival in the study group at 5 years post-CBT were $86.6 \%$ vs $66 \%$ for the control group [12].

\section{StemEx ${ }^{\circledast}$ copper chelator expansion}

Based on pre-clinical evidence that low concentrations of copper prevents stem cell differentiation in an in vitro culture system, Gamida Cell developed StemEx ${ }^{\circledR}$ which combines the copper chelator tetraethylenepentamine (TEPA) with early and late acting hematopoietic cytokines [13, 14]. The StemEx ${ }^{\circledR}$ UCB graft is derived from a single UCB unit. A fraction of the unit (contained in a separate segment of the cryobag) is thawed and expanded for 21 days in the presence of TEPA. On the day of transplantation, the recipient received the expanded fraction along with the unmanipulated fraction. StemEx ${ }^{\circledR}$ was first studied and found to be safe and feasible in a phase I/II trial performed at the MD Anderson Cancer Center [15].

Based on this early experience, Gamida Cell launched a multinational phase II/III registration trial designed to test the hypothesis that more rapid neutrophil engraftment facilitated by StemEx ${ }^{\circledR}$ would positively impact survival at 100 days following transplantation. The study accrued 101 subjects with high-risk leukemia and lymphoma from 25 stem cell transplant centers in the United States, Europe and Israel. Study patients were compared to a contemporaneous control group from the Center for International Blood and Marrow Transplant Research. The control population $(n=295)$ received myeloablative unmanipulated dual UCB transplantation between 2006 and 2010. Median nucleated cell and CD34+ were 400 and 77-fold expanded, respectively, with a mean total CD34+ infused of $9.7 \times 10^{5} / \mathrm{kg}$. The 100 -day survival was $84.2 \%$ for the study group versus $74.6 \%$ for the control group (odds ratio $0.50 ; 95 \% \mathrm{CI}$ $0.26-0.95 ; p=0.035$ ). Survival at day 180 was found to be similar for the 2 groups. Faster median neutrophil (21 days versus 28 days; $p<0.0001$ ), and platelet (54 days versus 105 days; $p=0.008$ ) engraftments were seen in the study group. Acute and chronic graft-versus-host disease rates were similar. The study suggests that transplanting expanded CD34+ stem cells from a portion of single UCB unit along with unmanipulated portion improved 100-day survival while improving neutrophil and platelet engraftments [16].

\section{NiCord $^{\circledR}$ expansion}

$\mathrm{NiCord}^{\circledR}$ is an ex vivo expanded cell product from an entire unit of umbilical cord blood that utilizes a small molecule, nicotinamide, as an epigenetic approach to inhibit differentiation and to increase the functionality of hematopoietic stem and progenitor cells expanded in ex vivo cultures. When nicotinamide is added to stimulatory hematopoietic cytokines, umbilical cord blood-derived hematopoietic progenitor cell cultures demonstrate an increased frequency of phenotypically primitive CD34+ CD38- cells. The cells also demonstrate increased migration toward stromal cell derived factor 1 . In a pre-clinical murine transplant model, they home to and engraft in the bone marrow with higher efficacy [17].

The unique feature of the $\mathrm{NiCord}^{\circledR} \mathrm{UCB}$ graft is that it consists of a hematopoietic stem-progenitor cell fraction that has been expanded for 21 days along with a non-cultured T-cell containing fraction that is collected and re-frozen following thaw. Thus, the $\mathrm{NiCord}^{\circledR}$ graft retains immunologic potency to augment both engraftment and immunologic recovery. 
The phase I study of $\mathrm{NiCord}^{\circledR}{ }^{\circledR}$ commenced in 2010 at Duke University in Durham North Carolina and Loyola University in Chicago Illinois. Eligible patients had highrisk hematologic malignancies and were candidates for dual umbilical cord blood transplantation. One unit was selected for NiCord ${ }^{\circledR}$ expansion and the other to be infused without manipulation. Recipients were conditioned with total body irradiation (1350 cGy) and fludarabine with or without cyclophosphamide. Key endpoint measures were compared to an historical control cohort of patients who received myeloablative conditioning followed by an unmanipulated dual UCB transplantation. Eleven patients received the NiCord ${ }^{\circledR}$ graft on this study. Full or partial neutrophil and T-cell engraftment derived from NiCord ${ }^{\circledR}$ was observed in eight patients. With a median follow-up of 24 months, $\mathrm{NiCord}^{\circledR}$ engraftment has remained stable in all patients. Two additional patients engrafted with the unmanipulated unit. Patients engrafting with $\mathrm{NiCord}^{\circledR}$ achieved significantly earlier neutrophil recovery (median of 11 days versus 25 days, $p=0.001$ ); and platelet recovery (30 versus 41 days, $p=0.012$ ), compared to controls [18].

This study confirms the presence of both long-term and short-term repopulating cells within the NiCord ${ }^{\circledR}$ expanded cord blood graft. Gamida Cell next launched a follow-up trial whereby recipients received myeloablative conditioning followed by a single NiCord ${ }^{\circledR}$ expanded graft (Clinicaltrials.gov NCT01816230). In this trial, the graft was cryopreserved following expansion and before delivery to the transplant center to address practical and logistical concerns surrounding the transport of a fresh cell therapy product. Thirty-six patients with hematologic malignancies were transplanted at 11 sites. The cumulative incidence of neutrophil engraftment at day 42 was $94 \%$. Two patients experienced secondary graft failure attributable to viral infections. Hematopoietic recovery was compared to that observed in recipients of standard UCB transplantation as reported to the CIBMTR $(n=146)$. The median time to neutrophil recovery was 11.5 days (95\% CI 9-14 days) for recipients of $\mathrm{NiCord}^{\circledR}$ and 21 days (95\% CI 20-23 days) for the comparator $(p<0.001)$. The median time to platelet recovery was 34 days (95\% CI 32-42 days) and 46 days (95\% CI 42-50 days) for the expanded and the comparator cohorts, respectively $(p<0.001)$ (Fig. 1). The cumulative incidence of grade II-IV acute GVHD at day 100 was $44 \%$, and grade III/IV acute GVHD at day 100 was $11 \%$. The cumulative incidence at 2 years of all chronic GVHD was $40 \%$, and moderate/severe chronic GVHD was $10 \%$. The 2-year cumulative incidences of non-relapse mortality and relapse were $24 \%$ and $33 \%$, respectively. The 2 -year probabilities of overall and disease-free survival were $51 \%$ and $43 \%$, respectively. The results showed that umbilical cord blood expanded ex vivo with nicotinamide provides more rapid neutrophil and platelet recovery compared to standard dual umbilical cord blood transplantation. Furthermore, NiCord appears safe, however longer follow-up will be required for confirmation. A long-term follow-up study is ongoing [19].

\section{Mesenchymal cell co-culture (mesoblast)}

Ex vivo expansion of unfractionated, previously cryopreserved umbilical cord blood is extremely challenging. Initiation of the culture with a purified population of CD34+ cells significantly enhances the expansion efficiency, but also adds to the complexity of the assay [20]. In order to avoid CD34+ cell selection, McNiece and colleagues developed a culture
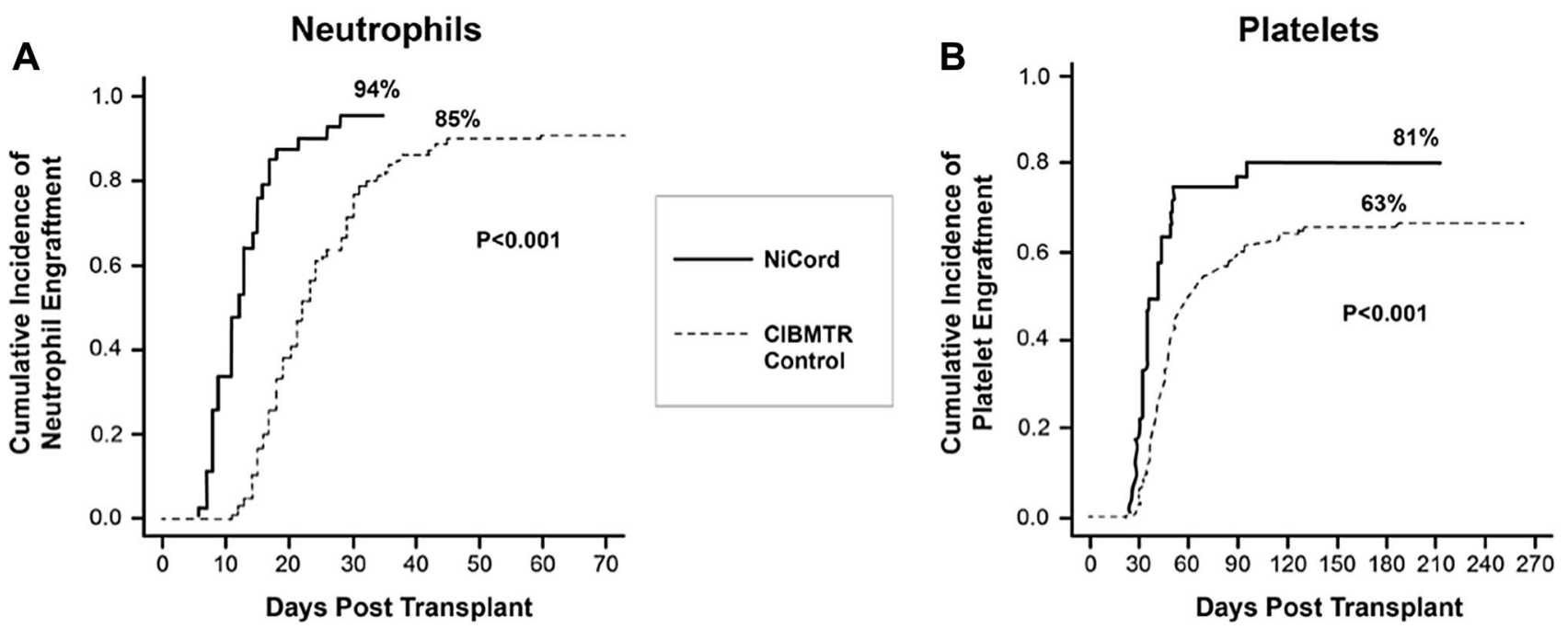

Fig. 1 Hematopoietic recovery. Weighted cumulative incidence of neutrophil by day 42 (a) and platelet recovery by day 100 (b) among recipients of NiCord ${ }^{\circledR}$ and a comparable retrospective cohort from the Center for International Blood and Marrow Transplant Research [19] 
system utilizing mesenchymal stomal cells (MSC) [21]. It is hypothesized that both contact-dependent and soluble factors provided by the MSCs more closely resemble the bone marrow micro-environment. Refinement of this technique ultimately led to development of a GMP-grade mesenchymal precursor product that was brought to the clinic by the group at MD Anderson Cancer Center. The 14-day UCB expansion process consists of co-culture with the adherent MSCs for 7 days in the presence of hematopoietic cytokines and an additional 7 days in the presence of cytokines only $[22,23]$.

Thirty-one adults with hematologic malignancies were treated with a dual UCB transplantation. The conditioning regimen was myeloablative, consisting of melphalan, thiotepa, fludarabine and rabbit anti-thymocyte globulin. One of the units was infused without manipulation and the other following expansion as described above. The outcome of these patients was compared to that of 80 historical controls who received a standard myeloablative dual UCB transplantation and whose outcome was reported to the Center for International Blood and Marrow Transplant Research data registry [24]. The cultured unit expanded by a median factor of 12.2 and CD34+ cells were expanded by a factor of 30.1. The expanded unit provided a median CD34+ cell dose of $0.95 \times 10^{6} / \mathrm{kg}$ recipient body weight. In comparison, the unmanipulated unit provided a median CD34+ cell dose of $0.38 \times 10^{6} / \mathrm{kg}$. The median time to neutrophil recovery was 15 days (range 9-42) and the median time to platelet recovery was 42 days (range 15-62). This compared favorably to the CIBMTR registry cohort who engrafted neutrophils on median day 24 (range 12-52) and platelets on median day 49 (range 18-264). Peripheral blood chimerism analysis performed in the peri-engraftment period demonstrated presence of the expanded unit in $46 \%$ of the subjects. By 6 months, the expanded unit was detectable in $13 \%$ of the subjects. By 1 year following transplantation, the unmanipulated unit in all the subjects provided hematopoiesis.

A 25-patient single-center phase II study combining cord blood expansion using mesenchymal stem cells and cord blood fucosylation (see Fucosylation technique) has been launched at the MD Anderson Cancer Center (Clinicaltrial. gov NCT03096782). By combining a stem and progenitor cell expansion technique with one that improves bone marrow homing, it is hoped that patients will experience prompt hematopoietic recovery. Patients with leukemia or lymphoma will be given 1 unit of expanded and fucosylated cord blood and 1 unit of non-expanded and non-fucosylated cord blood. Safety and count recovery will be studied.

\section{HSC835 aryl hydrocarbon}

Boitano and colleagues screened a library of 100,000 compounds resulting in identification of a purine derivative [StemRegenin 1 (SR1)] with marked ability to expand human CD34+ hematopoietic stem and progenitor cells ex vivo [25]. For this technology, a purified population of CD34+ cells is used to initiate the cell culture. During a 3 -week culture in serum-free media containing SR1 supplemented with thrombopoietin, stem cell factor, flt 3 ligand and interleukin-6, the CD34+ cells expand 1118-fold relative to input cells. Removal of SR1 from the culture system led to rapid differentiation, highlighting the importance of SR1 in the culture system. Cultured cells also produced high-level engraftment in immunodeficient mice. A 3-week expansion of 300 UCB CD34+ cells provided a comparable level of human engraftment in NSG mice as 10,000 uncultured UCB $\mathrm{CD} 34+$ cells. These results demonstrate that SR1 promotes expansion of UCB CD34+ cells that contribute to both early and sustained engraftment.

The phase I/II study enrolled 20 patients and 17 completed the treatment plan containing SR1 expanded UCB graft after myeloablative conditioning in the double UCBT setting. The unit with higher cell dose and HLA match was left unmanipulated while the second unit with lower cell dose was selected for ex vivo expansion. The expanded units had higher CD34+ cells with median of $17.5 \times 10^{6}$ cells per kilogram actual body weight while unmanipulated UCB units had $0.2 \times 10^{6}$ cells $/ \mathrm{kg}$. Study concluded that SR1 expansion culture reliably provides greater than 200 -fold expansion of CD34+ cells. For 17 patients who received study treatment, neutrophil recovery was median of 15 days (6-30) while $86 \%$ of patients in the control group recovered at median of 24 days. Platelet recovery was 49 days (28-136) vs 89 days, respectively. Hematopoiesis was principally derived from the expanded unit in 11 patients and unmanipulated unit in six. Neutrophil recovery was also more rapid with median of 11 days (6-23) in patients who engrafted with the expanded unit while it was 23 days (14-30) in those who engrafted with the unmanipulated unit. For those engrafting with the expanded UCB unit, the myeloid engraftment was durable with median follow-up of 272 days (35-688) [26].

Aryl-hydrocarbon receptor antagonist expanded UCB product, now called MGTA-456, was studied as a standalone graft after myeloablative conditioning (MAC) or non-myeloablative conditioning (NMAC). Twenty patients with high-risk hematologic malignancies and a partially HLA-matched UCB unit were enrolled. Ten were treated with cyclophosphamide (CY) $120 \mathrm{mg} / \mathrm{kg}$, fludarabine (FLU) $75 \mathrm{mg} / \mathrm{m}^{2}$ and TBI $1320 \mathrm{cGy}$ (MAC) and ten with CY $50 \mathrm{mg} / \mathrm{kg}$, FLU $200 \mathrm{mg} / \mathrm{m}^{2}$ and TBI $200 \mathrm{cGy}$ (NMAC) while all received GVHD prophylaxis with cyclosporine and mycophenolate mofetil. CD34+ cells expanded 324fold (range 42-1643). Outcomes were compared to similarly treated historical cohorts $(n=151 \mathrm{MAC} ; n=132$ NMAC). For recipients of MAC, MGTA-456 engrafted in all patients at a median of 14 days (range 7-32) as compared 
to $89 \%$ (median 23 days, range 19-31) in the control population $(p<0.01)$. For recipients of NMAC, MGTA-456 also engrafted in all patients at a median of 7 days (range 6-14) as compared to $94 \%$ engraftment (median 15 days, range 7-22) in control. Donor chimerism was found to be rapid and sustained. For recipients who received MAC followed by the MGTA-456 graft compared to the historical cohort, the incidence of grade 3-4 acute GVHD (aGVHD) was $22 \%$ vs. $24 \%$, respectively; chronic GVHD (cGVHD), $11 \%$ vs. $21 \%$, respectively; transplant-related mortality (TRM), $11 \%$ vs. $34 \%$, respectively; and overall survival (OS), $67 \%$ vs $55 \%$, respectively. For NMAC patients, results were similar except for a higher risk of aGVHD in recipients of MGTA456 (aGVHD 3-4, 43\% vs $15 \%$; cGVHD, $0 \%$ vs $19 \%$; TRM, $22 \%$ vs $20 \%$; and OS, $44 \%$ vs $49 \%$ ), respectively [27].

\section{UM171}

UM171 is one of the newly synthesized analogs of UM729 which is a low-molecular-weight compounds that were found to expand human CD34+ CD45RA- mobilized peripheral blood cells that are enriched in long-term repopulating hematopoietic stem cells. UM171 was 10-20 times more potent than UM729 in expansion of human CD34+ CD45RA- cells. Fares et al. in 2014 suggested that UM729 did not suppress aryl-hydrocarbon receptor pathway but instead enhances the human long-term hematopoietic stem cell self-renewal process [28].

To further elucidate the mechanistic pathway, in their 2017 paper in Blood, Fares et al. showed that endothelial protein C receptor (EPCR) positive UM171-treated cells, compared with EPCR negative cells, showed robust multilineage repopulation and serial reconstitution in immunocompromised mice [29].

In a phase I/II clinical trial that recently completed recruitment at Maisonneuve-Rosemont Hospital in Canada, 25 patients with high-risk leukemia were enrolled to receive UM171 expanded cord blood cells to establish safety and feasibility. Patients will be followed for 3 years (Clinicaltrials.gov NCT02668315).

Separately, a new phase I/II trial at Ciusss de L'Est de l'Île de Montréal started recruiting 20 multiple myeloma patients to evaluate safety and efficacy of UM171 in multiple myeloma (Clinicaltrial.gov NCT03441958). Newly diagnosed multiple myeloma patients with high-risk disease without $6 / 6$ compatible sibling donor will be evaluated before or during autologous stem cell transplant. After a bortezomib-based induction for a minimum of 4 cycles, followed by Melphalan $>140 \mathrm{mg} / \mathrm{m}^{2}$ and auto transplant, eligible patients will undergo screening evaluation. Once eligible, patients will receive conditioning regimen before receiving outpatient non-myeloablative allogeneic HSCT with UM171 expanded CB on day 0.

\section{Prostaglandin e2 co-culture}

The previously discussed technologies are aimed at addressing the low stem cell content of the UCB by ex vivo expansion of both short-term and long-term hematopoietic progenitor cells. In contrast, the prostaglandin e2 co-culture methodology was conceived as an inexpensive, safe and practical method for augmenting the homing and engraftment potential of UCB stem cells without expansion. The method utilizes 16,16-dimethyl prostaglandin E2 (dmPGE2) previously identified in a zebra fish stem cell model to be a critical regulator of hematopoietic stem cell homeostasis [30]. From this pre-clinical work, it was hypothesized that brief ex vivo exposure of UCB to dmPGE2 could improve patient outcomes by increasing the "effective dose" of hematopoietic stem cells without significant toxicity. This hypotheses was tested in a phase I study conducted at the Dana Farber Cancer Institute in Boston. The investigators enrolled recipients of non-myeloablative dual UCB transplantation. One unit was infused following a brief exposure to dmPGE2 and the other was infused without manipulation. Two cohorts of patients were enrolled. In cohort 1 , the investigational unit was incubated with $10 \mathrm{mM}$ of dmPGE2 for $60 \mathrm{~min}$ at $4{ }^{\circ} \mathrm{C}$ ( 9 patients). Cohort 2 consisted of 12 patients in whom the larger of the 2 UCB units was incubated with the same concentration dmPGE2, but for 120 min instead of $60 \mathrm{~min}$ and at $37^{\circ} \mathrm{C}$ instead of $4{ }^{\circ} \mathrm{C}$.

While patients in cohort 1 experienced no safety concerns, there was no demonstrable benefit of the dmPGE2 treated unit. However, the optimized co-culture conditions utilized for patients in cohort 2 resulted in more rapid median time to neutrophil engraftment compared to historical controls ( 17.5 days vs. 21 days, $p=0.45$ ). Furthermore, 10 of 12 patients showed complete and sustained engraftment of the dmPGE2-treated units. While it is possible the engraftment advantage afforded to the manipulated unit is a consequence of it being the larger of the two infused grafts, it is also possible that co-culture with PGE2 did indeed improve homing of the resident stem cells. No safety concerns emerged from this small phase I trial [31]. Based on promising results emerging from this trial, a randomized phase II trial supported by Fate Therapeutics (Clinicaltrials. gov NCT01627314) was initiated in 2012 [22, 23] Unfortunately, the trial was terminated in 2017 due to poor accrual.

\section{C3a complement co-culture}

Complement fragment $3 \mathrm{a}(\mathrm{C} 3 \mathrm{a})$ is a product of proteolytic cleavage of complement protein $\mathrm{C} 3$. Along with a myriad of immunoregulatory properties, $\mathrm{C} 3 \mathrm{a}$ also sensitizes human hematopoietic stem and progenitor cells to homing properties of stromal derived factor 1 (SDF-1) through binding of the $\mathrm{C} 3 \mathrm{a}$ receptor present on the cell surface. Pre-clinical 
murine transplant models demonstrated that incubation of hematopoietic stem cells with $\mathrm{C} 3$ a prior to transplantation into lethally irradiated hosts accelerates engraftment kinetics $[32,33]$.

Based on these promising pre-clinical data, a phase I/ II study was performed at the University of Minnesota to assess safety and efficacy of $\mathrm{C} 3 \mathrm{a}$ in promoting engraftment of UCB stem cells [34]. Adults receiving non-myeloablative dual umbilical cord blood transplantation were eligible for the trial. One of the 2 UCB grafts was incubated at room temperature with GMP-grade $\mathrm{C} 3$ a prior to transplantation. Twenty-nine patients were treated on the study. Mild infusion-related side effects were observed following infusion of the C3a-primed units. Because the patients were conditioned with a non-myeloablative regimen and therefore experienced early autologous reconstitution, the impact of C3a on engraftment kinetics could not be assessed. Of the 27 patients who achieved neutrophil engraftment, 9 had hematopoiesis derived from the $\mathrm{C} 3 \mathrm{a}$ primed unit and 18 from the unmanipulated unit. Thus, C3a treatment did not appear to provide an engraftment advantage [22, 23].

\section{Fucosylation}

Like dmPGE2 and C3a co-culture, ex vivo fucosylation is a technique under development that is designed to augment homing of umbilical cord blood stem cells to the bone marrow stroma. The rationale for this strategy hinges on the fact that UCB stem cells do not home as avidly to the bone marrow as those from adult bone marrow or mobilized peripheral blood. This defect in bone marrow homing may, in part, be due to lack of binding to the critical adhesion molecules P- and E-selectin which are expressed on bone marrow endothelial cells [35]. Fucosylation of selectin ligands expressed on UCB stem cells increases affinity to $\mathrm{P}$ - and E-selectin. Thus, in pre-clinical models of UCB transplantation, engraftment of UCB stem cells into immunodeficient mice can be improved with pre-transplant ex vivo fucosylation [36, 37].

The group at MD Anderson showed that endogenously fucosylated cord blood hematopoietic and stem progenitor cells engraft more efficiently in a xenogeneic transplantation model than non-fucosylated HSPCs. They suggest that the majority of endogenously non-fucosylated CB HSPCs are a good target for enforced fucosylation to improve engraftment following cord blood transplantation [38].

Phase I testing of this technique was reported by the group from MD Anderson [39]. Twenty-two patients with high-risk hematologic malignancies received myeloablative bone marrow conditioning followed by transplantation with two UCB units. One unit was infused without manipulation while the other was incubated for $30 \mathrm{~min}$ in the presence of guanosine diphosphate fucose along with the enzyme
fucosyltransferase-VI, a technique shown to improve cell surface fucosylation of hematopoietic stem cells. Engraftment kinetics were compared to an historical cohort transplanted in a similar fashion with two unmanipulated UCB units. A statistically significant reduction in time to neutrophil and platelet engraftment was observed in recipients of a fucosylated graft (17 versus 26 days, $p=0.0003$ and 35 versus 45 days, $p=0.0065$, respectively). Interestingly, faster blood count recovery was not dependent on engraftment or dominance of the treated unit, which occurred only 9 of the 22 subjects. This suggests that both units following infusion share beneficial effects of fucosylation [22, 23].

\section{Long-term immune recovery}

Recovery of immune function following transplantation of ex vivo expanded cord blood is of great interest, but to date the published data on this topic is limited. Anand et al. performed a single-center study of comparing infectious complications during the first 100 days following transplantation of expanded versus unmanipulated cord blood stem cells. They found a significant reduction in clinically significant bacterial infections. However, there was no observed difference in the viral and fungal infection rate [40]. In a phase $1 / 2$ international multicenter trial, 27 NiCord recipients were compared with 27 unmanipulated cord blood transplant patients and $20 \mathrm{~T}$-depleted unrelated bone marrow transplant patients. NiCord transplant recipients were adults while the comparator cohorts were pediatric (NiCord median age 41.5 years, unmanipulated cord blood transplant cohort with median age 15.4 and unrelated BMT cohort with median age 14.3). Despite the age difference, similar reconstitution of $T$ cells, monocytes, conventional and plasmacytoid dendritic cells were observed. Reconstitution of NK cells, B cells and plasma cells was faster after NiCord HCT, compared to two other cohorts [41].

\section{Conclusion}

In the early 2000s, the advantages of UCB transplantation were only being appreciated in the pediatric population where outcomes were comparable to that of adult unrelated donor transplantation. Since then, a better understanding of the minimum safe transplantable cell dose, improved quality and characterization of the UCB inventory, and the advent of dual UCB transplantation has broadened the applicability to the adult population. However, limitations remain and chief among them is the kinetics of hematopoietic recovery following UCB transplantation. Ex vivo manipulation of the UCB graft prior to transplantation holds great promise in addressing these limitations. Clinical data are already available from multiple techniques demonstrating more rapid 
neutrophil and platelet recovery. Short-term safety has also been demonstrated. Confirmation of long-term safety and reduction in the graft failure rate will require longer followup and larger studies. With the development of improved techniques for the transplantation of haplo-identical related donor grafts, the major challenge going forward will be demonstration of cost effectiveness and practicality of ex vivo UCB stem cell manipulation.

\section{Compliance with ethical standards}

Conflict of interest Ko Maung does not report any conflict of interest. He is supported by NIH T32HL007057 grant. Mitchell Horwitz has received research support from, and has served as a consultant for Gamida Cell Ltd.

\section{References}

1. Peffault de Latour R, Brunstein CG, Porcher R, Chevallier P, Robin M, Warlick E, et al. Similar overall survival using sibling, unrelated donor, and cord blood grafts after reduced-intensity conditioning for older patients with acute myelogenous leukemia. Biol Blood Marrow Transplant. 2013;19(9):1355-60.

2. Smith AR, Baker KS, Defor TE, Verneris MR, Wagner JE, Macmillan ML. Hematopoietic cell transplantation for children with acute lymphoblastic leukemia in second complete remission: similar outcomes in recipients of unrelated marrow and umbilical cord blood versus marrow from HLA matched sibling donors. Biol Blood Marrow Transplant. 2009;15(9):1086-93.

3. Jaroscak J, Goltry K, Smith A, Waters-Pick B, Martin PL, Driscoll TA, et al. Augmentation of umbilical cord blood (UCB) transplantation with ex vivo-expanded UCB cells: results of a phase 1 trial using the AastromReplicell System. Blood. 2003;101(12):5061-7.

4. Scaradavou A, Brunstein CG, Eapen M, Le-Rademacher J, Barker JN, Chao N, et al. Double unit grafts successfully extend the application of umbilical cord blood transplantation in adults with acute leukemia. Blood. 2013;121(5):752-8.

5. Barker JN, Weisdorf DJ, DeFor TE, Blazar BR, McGlave PB, Miller JS, et al. Transplantation of 2 partially HLA-matched umbilical cord blood units to enhance engraftment in adults with hematologic malignancy. Blood. 2005;105(3):1343-7.

6. Milner LA, Kopan R, Martin DI, Bernstein ID. A human homologue of the Drosophila developmental gene, Notch, is expressed in CD34+ hematopoietic precursors. Blood. 1994;83(8):2057-62.

7. Delaney C, Varnum-Finney B, Aoyama K, Brashem-Stein C, Bernstein ID. Dose-dependent effects of the Notch ligand Delta1 on ex vivo differentiation and in vivo marrow repopulating ability of cord blood cells. Blood. 2005;106(8):2693-9.

8. Delaney C, Heimfeld S, Brashem-Stein C, Voorhies H, Manger RL, Bernstein ID. Notch-mediated expansion of human cord blood progenitor cells capable of rapid myeloid reconstitution. Nat Med. 2010;16(2):232-6

9. Gutman JA, Turtle CJ, Manley TJ, Heimfeld S, Bernstein ID, Riddell SR, et al. Single-unit dominance after double-unit umbilical cord blood transplantation coincides with a specific CD8+ T-cell response against the nonengrafted unit. Blood. 2010;115(4):757-65.

10. Moretta A, Andriolo G, Lisini D, Martinetti M, Pasi A, Rebulla $\mathrm{P}$, et al. In vitro evaluation of graft-versus-graft alloreactivity as a tool to identify the predominant cord blood unit before double cord blood transplantation. Biol Blood Marrow Transplant. 2012;18(7):1108-18.

11. Delaney C, Milano F, Shelly H, Nicoud I, Bernstein ID. Infusion of non-hla matched, off-the-shelf ex vivo expanded cord blood progenitor cells in patients undergoing myeloablative cord blood transplantation is safe and decreases the time to neutrophil recovery. Biol Blood Marrow Transplant. 2012;18(2):S203.

12. Milano F, Cox E, Heimfeld S, Bernstein I, Applebaum F, Delaney C. Non-HLA matched, ex-vivo expanded cord blood product significantly improves kinetics of hematopoietic recovery and results in excellent survival in patients undergoing cord blood transplantation. In: EHA congress; 15 June 2018. 2018.

13. Peled T, Landau E, Mandel J, Glukhman E, Goudsmid NR, Nagler A, et al. Linear polyamine copper chelator tetraethylenepentamine augments long-term ex vivo expansion of cord blood-derived CD34+ cells and increases their engraftment potential in NOD/ SCID mice. Exp Hematol. 2004;32(6):547-55.

14. Peled T, Mandel J, Goudsmid RN, Landor C, Hasson N, Harati $\mathrm{D}$, et al. Pre-clinical development of cord blood-derived progenitor cell graft expanded ex vivo with cytokines and the polyamine copper chelator tetraethylenepentamine. Cytotherapy. 2004;6(4):344-55.

15. de Lima M, McMannis J, Gee A, Komanduri K, Couriel D, Andersson BS, et al. Transplantation of ex vivo expanded cord blood cells using the copper chelator tetraethylenepentamine: a phase I/II clinical trial. Bone Marrow Transplant. 2008.

16. Stiff PJ, Montesinos P, Peled T, Landau E, Goudsmid NR, Mandel J, Hasson N, Olesinski E, Glukhman E, Snyder DA, Cohen EG, Kidron OS, Bracha D, Harati D, Ben-Abu K, Freind E, Freedman LS, Cohen YC, Olmer L, Barishev R, Rocha V, Gluckman E, Horowitz MM, Eapen M, Nagler A, Sanz G. Cohort-controlled comparison of umbilical cord blood transplantation using carlecortemcel-L, a single progenitor-enriched cord blood, to double cord blood unit transplantation. Biol Blood Marrow Transplant. 2018;24(7):1463-70.

17. Peled T, Shoham H, Aschengrau D, Yackoubov D, Frei G, Rosenheimer GN, et al. Nicotinamide, a SIRT1 inhibitor, inhibits differentiation and facilitates expansion of hematopoietic progenitor cells with enhanced bone marrow homing and engraftment. Exp Hematol. 2012;40(4):342-355 e1.

18. Horwitz ME, Chao NJ, Rizzieri DA, Long GD, Sullivan KM, Gasparetto C, et al. Umbilical cord blood expansion with nicotinamide provides long-term multilineage engraftment. J Clin Invest. 2014;124(7):3121-8.

19. Horwitz ME, Wease S, Blackwell B, Valcarcel D, Frassoni F, Boelens JJ, Nierkens S, Jagasia M, Wagner JE, Kuball J, Koh LP, Majhail NS, Stiff PJ, Hanna R, Hwang WYK, Kurtzberg J, Cilloni D, Freedman LS, Montesinos P, Sanz G. Nicotinamide-expanded single UCB transplantation. J Clin Oncol 2019 (in press).

20. Briddell RA, Hartley CA, Smith KA, McNiece IK. Recombinant rat stem cell factor synergizes with recombinant human granulocyte colony-stimulating factor in vivo in mice to mobilize peripheral blood progenitor cells that have enhanced repopulating potential. Blood. 1993;82(6):1720-3.

21. McNiece I, Harrington J, Turney J, Kellner J, Shpall EJ. Ex vivo expansion of cord blood mononuclear cells on mesenchymal stem cells. Cytotherapy. 2004;6(4):311-7.

22. Horwitz ME, Frassoni F. Improving the outcome of umbilical cord blood transplantation through ex vivo expansion or graft manipulation. Cytotherapy. 2015;17(6):730-8.

23. Horwitz ME. Ex vivo expansion or manipulation of stem cells to improve outcome of umbilical cord blood transplantation. Curr Hematol Malig Rep. 2016;11(1):12-8.

24. de Lima M, McNiece I, Robinson SN, Munsell M, Eapen M, Horowitz M, et al. Cord-blood engraftment with ex vivo mesenchymal-cell coculture. N Engl J Med. 2012;367(24):2305-15. 
25. Boitano AE, Wang J, Romeo R, Bouchez LC, Parker AE, Sutton SE, et al. Aryl hydrocarbon receptor antagonists promote the expansion of human hematopoietic stem cells. Science. 2010;329(5997):1345-8.

26. Wagner JE, Brunstein CG, Boitano AE, DeFor TE, McKenna D, Sumstad D, Blazar BR, Tolar J, Le C, Jones J, Cooke MP, Bleul CC. Phase I/II trial of stemregenin-1 expanded umbilical cord blood hematopoietic stem cells supports testing as a stand-alone graft. Cell Stem Cell. 2016;18(1):144-55.

27. Wagner JE, Brunstein CG, Defor TE, Boitano AE, McKenna DH, Sumstad D, Sanna B, Bleul CC, Cooke M. Single cord blood units (CBU) expanded with an aryl hydrocarbon receptor (AHR) antagonist, demonstrate uniform engraftment and rapid hematopoietic recovery. In: BMT tandem meetings; Salt Lake City. 2018.

28. Fares I, Chagraoui J, Gareau Y, Gingras S, Ruel R, Mayotte N, Csaszar E, Knapp DJ, Miller P, Ngom M, Imren S, Roy DC, Watts KL, Kiem HP, Herrington R, Iscove NN, Humphries RK, Eaves CJ, Cohen S, Marinier A, Zandstra PW, Sauvageau G. Pyrimidoindole derivatives are agonists of human hematopoietic stem cell self-renewal. Science. 2014;345(6203):1509-12.

29. Fares I, Chagraoui J, Lehnertz B, MacRae T, Mayotte N, Tomellini E, Aubert L, Roux PP, Sauvageau G. EPCR expression marks UM171-expanded CD34+ cord blood stem cells. Blood. 2017;129(25):3344-51.

30. North TE, Goessling W, Walkley CR, Lengerke C, Kopani KR, Lord AM, et al. Prostaglandin E2 regulates vertebrate haematopoietic stem cell homeostasis. Nature. 2007;447(7147):1007-11.

31. Cutler C, Multani P, Robbins D, Kim HT, Le T, Hoggatt J, et al. Prostaglandin-modulated umbilical cord blood hematopoietic stem cell transplantation. Blood. 2013;122(17):3074-81.

32. Ratajczak J, Reca R, Kucia M, Majka M, Allendorf DJ, Baran JT, et al. Mobilization studies in mice deficient in either $\mathrm{C} 3$ or $\mathrm{C} 3 \mathrm{a}$ receptor $(\mathrm{C} 3 \mathrm{aR})$ reveal a novel role for complement in retention of hematopoietic stem/progenitor cells in bone marrow. Blood. 2004;103(6):2071-8.

33. Reca R, Mastellos D, Majka M, Marquez L, Ratajczak J, Franchini $\mathrm{S}$, et al. Functional receptor for $\mathrm{C} 3 \mathrm{a}$ anaphylatoxin is expressed by normal hematopoietic stem/progenitor cells, and C3a enhances their homing-related responses to SDF-1. Blood. 2003;101(10):3784-93.

34. Brunstein CG, McKenna DH, DeFor TE, Sumstad D, Paul P, Weisdorf DJ, et al. Complement fragment 3a priming of umbilical cord blood progenitors: safety profile. Biol Blood Marrow Transplant. 2013;19(10):1474-9.
35. Hidalgo A, Weiss LA, Frenette PS. Functional selectin ligands mediating human $\mathrm{CD} 34(+)$ cell interactions with bone marrow endothelium are enhanced postnatally. J Clin Invest. 2002;110(4):559-69.

36. Robinson SN, Thomas MW, Simmons PJ, Lu J, Yang H, Parmar S, et al. Fucosylation with fucosyltransferase VI or fucosyltransferase VII improves cord blood engraftment. Cytotherapy. 2014;16(1):84-9.

37. Xia L, McDaniel JM, Yago T, Doeden A, McEver RP. Surface fucosylation of human cord blood cells augments binding to $\mathrm{P}$-selectin and E-selectin and enhances engraftment in bone marrow. Blood. 2004;104(10):3091-6.

38. Robinson SN, Thomas MW, Simmons PJ, Lu J, Yang H, Javni JA, Shpall EJ, Zweidler-Mckay PA. Non-fucosylated CB CD34+ cells represent a good target for enforced fucosylation to improve engraftment following cord blood transplantation. Cytotherapy. 2017;19(2):285-92.

39. Popat U, Mehta RS, Rezvani K, Fox P, Kondo K, Marin D, et al. Enforced fucosylation of cord blood hematopoietic cells accelerates neutrophil and platelet engraftment after transplantation. Blood. 2015;125(19):2885-92.

40. Anand S, Thomas S, Hyslop T, Adcock J, Corbet K, Gasparetto C, Lopez R, Long GD, Morris AK, Rizzieri DA, Sullivan KM, Sung KD, Sarantopoulos S, Chao NJ, Horwitz ME. Transplantation of ex vivo expanded umbilical cord blood (NiCord) decreases early infection and hospitalization. Biol Blood Marrow Transplant. 2017;23:1151-7.

41. de Koning C, Horwitz ME, Sanz G, Jagasia M, Wagner JE, Stiff PJ, Hanna R, Cilloni D, Boelens JJ, Nierkens S. Rapid and robust CD4+ and CD8+ T-, NK-, B-cell, dendritic cell, and monocyte reconstitution after nicotinamide-expanded cord blood transplantation. In: Transplantation \& cellular therapy of ASBMT and CIBMTR; 20-24 February 2019; Houston. 2019.

42. Robinson SN, Simmons PJ, Thomas MW, Brouard N, Javni JA, Trilok S, et al. Ex vivo fucosylation improves human cord blood engraftment in NOD-SCID IL-2Rgamma(null) mice. Exp Hematol. 2012;40(6):445-56.

Publisher's Note Springer Nature remains neutral with regard to jurisdictional claims in published maps and institutional affiliations. 